\title{
Impact of Longus Colli Muscle Massage on the Strength and Endurance of the Deep Neck Flexor Muscle of Adults
}

\author{
Wontae Gong, $\mathrm{PhD}, \mathrm{PT}^{1)}$ \\ 1) Department of Physical Therapy, Korea Nazarene University: Wolbong Ro 48, Seobuk-gu, \\ Cheonan-si, Chungcheongnam-do 330-718, Republic of Korea. TEL: +82 41-570-4286, \\ FAX: +82 41-570-7925
}

\begin{abstract}
Purpose] The purpose of the present study was to examine the effects of longus colli muscle massage on the strength and endurance of the deep neck flexor muscle in adults. [Subjects] A total of 60 subjects were divided into an experimental group of 30 subjects and a control group of 30 subjects. [Methods] The experimental group received massage of the longus colli muscle, which is the deep neck flexor muscle, and the control group received superficial neck muscle massage. The strength and endurance of both the experimental group and the control group were measured before and after the intervention using a pressure biofeedback unit (PBU). [Results] After the experiment, the strength of DNF of the experimental group showed a statistically significant increase, and the endurance of DNF of the experimental group showed an increase in its average value. The independent sample ttest revealed no statistically significant differences in the groups. [Conclusion] Massage of the longus colli muscle, which is the deep neck flexor, was shown to improve its strength and endurance, which are measures of neck stabilization. Therefore, longus colli muscle massage can be performed for patients who cannot perform neck-stabilizing exercises or before performing other neck-stabilizing exercises.
\end{abstract}

Key words: Deep neck flexor, Longus colli, Massage

(This article was submitted Dec. 11, 2012, and was accepted Dec. 29, 2012)

\section{INTRODUCTION}

In the daily lives of most modern people, the time spent working with the neck bent forward, working on a computer, riding in cars, and engaging in leisure activities while in a sitting positions has been increasing. This sitting causes overloads in the soft tissues that support the neck, and repeated damage adversely affects neck stability. Therefore, understanding neck-stabilizing exercises is necessary not only to prevent neck diseases but also to reduce chronic neck pain. Recently, among therapeutic exercises, the importance of stabilizing exercises that strengthen deep muscles, such as the longus colli muscle and the longus capitis muscle, which play leading roles in maintaining the stability of the spine in order to keep the cervical spine in the neutral position, has been noticed.

Many previous studies have reported that stabilizing exercises are quite effective at reducing chronic neck pain and improving neck function ${ }^{1)}$. Previous studies have also reported that neck-stabilizing exercises greatly affected the strength and endurance of deep muscles. Among these stabilizing exercise methods, a method using slings ${ }^{2}$ and a method using cervical flexion exercises ${ }^{3)}$ and a PBU in the supine position ${ }^{4}$ have been reported. Recently, neck-stabilizing exercises that can be performed in standing positions have been developed, so that neck-stabilizing exercises can

E-mail: owntae@hanmail.net be performed anytime, anywhere ${ }^{5}$.

However, all these methods require patients' active participation. Reports in which massage, a passive method, was directly applied to the longus colli muscle and its effect on the strength and endurance of the DNF are rare. Patient with acute pain cannot perform stabilizing exercises, and elderly persons with low cognitive abilities cannot improve their neck stabilization abilities through stabilizing exercises that require active participation.

The present study directly applied massage to the longus colli muscle, which is an important element in neck stabilization, and examined its effect of the massage on the strength and endurance of the deep muscles, important elements in neck stabilization.

\section{SUBJECTS AND METHODS}

The subjects who participated in the present study were 60 students of N University in Korea. They were divided into an experimental group of 30 subjects $(15$ males and 15 females) and a control group of 30 subjects (15 males and 15 females). The experimental group's age was $23.1 \pm 3.2$ years, its height was $166.4 \pm 7.9 \mathrm{~cm}$, and its weight was $62.7 \pm 11.7 \mathrm{~kg}$. The control group's age was $23.1 \pm 4.3$ years, its height was $166.3 \pm 7.2 \mathrm{~cm}$, and its weight was $61.6 \pm 10.3 \mathrm{~kg}$. There were no statistically significant difference between the two groups in age, height or weight $(p>0.05)$. All the subjects were adults aged at least 20 years. Those who had under- 


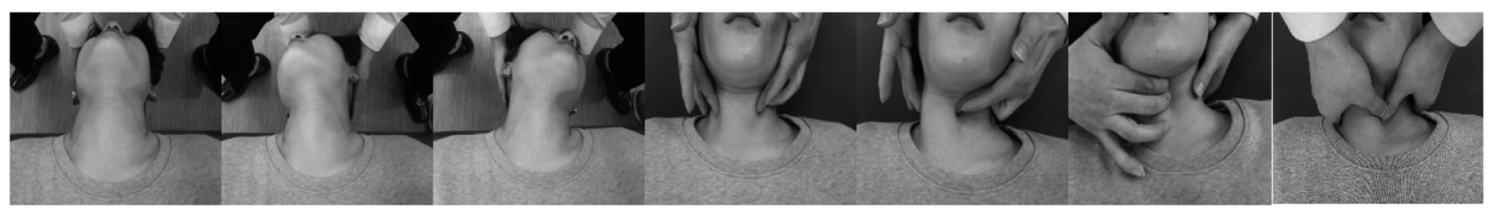

Fig. 1. Longus colli massage process (The throat and bronchial tubes were pushed to one side using a finger. Ischemic compression applied to the longus colli)

Table 1. Comparison of strength and endurance with-in pre-test and post-test in each group (mean $\pm \mathrm{SD}$ ) (unit: sec)

\begin{tabular}{lccc}
\hline Category & & Pre-test & Post-test \\
\hline \multirow{2}{*}{ Experiment group } & Strength* & $22.6 \pm 22.6$ & $38.5 \pm 23.0$ \\
& Endurance & $48.9 \pm 24.1$ & $71.8 \pm 28.4$ \\
\multirow{2}{*}{ Control group } & Strength & $19.4 \pm 19.6$ & $21.7 \pm 21.3$ \\
& Endurance & $52.4 \pm 29.8$ & $51.9 \pm 29.0$ \\
\hline
\end{tabular}

$* \mathrm{p}<0.05$

gone surgery, those who were receiving treatment at a hospital, and those who had ruptured cervical disks were excluded from the study. The purpose of the study and the entire process of the experiment were explained to all the subjects, and voluntary written agreements were received from all of them before they participated in the experiment.

The experimental group was given massage in order to relax the longus colli muscle, while the control group was given general neck massage. After applying the massage once, the immediate effect was tested. In the experimental group, for massage of the longus colli muscle, the subjects first lay down on massage table in the supine position and with $\mathrm{C} 7$ placed on the table, and C6 off the table in order to induce cervical spine extension. At this time, the subject's head was supported by the hands of the therapist in order to induce slow extension. Thereafter, in order to find out the tonus of the longus colli muscle, extension was induced to the left and to the right, while $15^{\circ}$ of cervical rotation was maintained. Then, massage was applied to the longus colli muscle on the side that was thought to have high muscle tone, between the left and right longus colli muscles, in order to relax this muscle. In this technique, the practitioner first checked the flexibility of the subject's throat and bronchial tubes by pushing the respective parts from left to right using the index and middle fingers of both hands. Then, in order to relax a particular side of the longus colli, the practitioner pushed the throat and bronchial tubes in the opposite direction, while applying a horizontal force so as to have the longus colli exposed. After this, the practitioner fixed his/her index and middle fingers on the subject's throat and bronchial tubes in a hook shape, and pressed the tense area of the longus colli with the tip of the thumb of the opposite hand in the dorsal direction; then gently pressed again, by placing the opposite thumb over the first thumb on the area. At this time, the therapist compressed the muscle at strength that would not cause tonus of the superficial muscles while sensing the relaxation of the longus colli muscle with the fingertips (Fig. 1). The longus colli massage was mainly applied to segments around C5, where shortening occurs. Each compression was maintained for approximately 5-7 seconds, and the compression was repeated 5-6 times or until muscle relaxation was felt by the therapist's fingertips. After massage, relaxation was checked through retests $^{6}$. For the control group, massage was applied to the sternocleidomastoid muscle, the superior trapezius muscle, the musculus levator scapulae, and the posterior muscles of the neck ${ }^{7}$.

The strength and endurance of the DNF were measured via revised cranio-cervical flexion tests (CCFT) using a PBU (Chattanooga Group, Australia). Strength was measured as the time which the maximum voluntary contractile strength (MVCS), the pressure of the maximum push over a base pressure of $80 \mathrm{mmHg}$, could be maintained, and Endurance was measured as the time during which the intermediate pressure between the base pressure and MVCS could be maintained ${ }^{5}$.

The experimental results were statistically analyzed using SPSS 12.0 KO (SPSS, Chicago, IL, USA). After the general characteristics of the subjects were determined, the paired t-test was used to compare the changes in strength and endurance that occurred between pre-test and posttest in each group. The differences between the two groups were tested using the independent t-test. The statistical significance level, $\alpha$, was set at 0.05 .

\section{RESULTS}

The strength and endurance of the experimental group and the control group before and after the experiment were compared. The strength of the experimental group showed a statistically significant difference between before and after the intervention, and the endurance of the experimental group showed an increase, although the increase was not statistically significant (Table 1). The independent sample ttest was conducted to test the significance of the differences in the pre-test, the post-test, and the differences between the pre-test and post-test values of the strength and endurance between the two groups; none of the differences were 
Table 2. Comparison of strength and endurance between experiment group and control group (mean $\pm \mathrm{SD}$ ) (unit: $\mathrm{sec}$ )

\begin{tabular}{lccc}
\hline Category & & Experiment group & Control group \\
\hline \multirow{2}{*}{ Pre-test } & strength & $22.6 \pm 22.6$ & $19.4 \pm 19.6$ \\
& endurance & $48.9 \pm 24.1$ & $52.7 \pm 29.8$ \\
\multirow{2}{*}{ Post-test } & strength* & $38.5 \pm 23.0$ & $21.7 \pm 21.3$ \\
& endurance & $71.8 \pm 28.4$ & $51.8 \pm 29.0$ \\
\multirow{2}{*}{ Difference } & strength & $15.9 \pm 13.7$ & $2.3 \pm 7.6$ \\
& endurance & $22.9 \pm 21.3$ & $-0.6 \pm 6.7$ \\
\hline
\end{tabular}

difference: difference of pre-test and post-test * $\mathrm{p}<0.05$

statisticaly significant (Table 2).

\section{DISCUSSION}

In the present study, massage was directly applied to the longus colli muscle, which plays an important role in neck stabilization, and the effects of the massage on the strength and endurance of the DNF were examined. Among previous studies, there are reports of massage directly applied to the longus colli muscle resulting in increases in the range of motion of the cervical spine ${ }^{6}$. However, no previous studies in which massage was directly applied to the longus colli muscle, as was done in the present study, could be found. Among the studies that reported improvement in the strength and endurance of the longus colli muscle after exercises, Jeon et al. reported improvement in the strength and endurance of subjects who performed cervical stabilizing exercises in either the standing or supine positions ${ }^{5)}$. Jull et al. conducted craniocervical flexion exercises and cervical flexion exercises for chronic neck pain patients for seven weeks, aiming to improve the strength and endurance of the cervical flexor muscle, and reported, the strength of the cervical flexor muscle was improved ${ }^{8)}$.

In the present study, massage was directly applied to the longus colli muscle, and a statistically significant, increase in muscle strength after the experiment was found in the experimental group. This result is considered have been the result of muscle relaxation induced by direct ischemic compression of the longus colli muscle after the bronchus and the esophagus had been pushed to one side. However, although endurance showed an increase in the average value, the increase was not statistically significant. The independent t-test did not find statisticaly significant differences between the grops, indicating that exercises are more effec- tive than massage at improving the strength and endurance of the longus colli muscle. However, because patients with acute pain or elderly persons with low cognitive abilities cannot actively participate in neck-stabilizing exercises, massage could be applied to the longus colli muscle, which is the deep neck flexor muscle, as an alternative method to improve strength and endurance, which are measures of neck stabilization. Therefore, longus colli muscle massage can be applied to patients who cannot perform neck-stabilizing exercises or before other neck-stabilizing exercises are performed.

\section{REFERENCES}

1) Ferreira PH, Ferreira ML, Maher CG, et al.: Specific stabilisation exercise for spinal and pelvic pain: a sysematic review. Aust J Physiother, 2006, 52: 79-88. [Medline] [CrossRef]

2) Kirkesola G: The Neurac method. Fysioterapeuten, 2009, 12: 16-25.

3) Grimmer K, Trott P: The association between cervical excursion angles and cervical short flexor muscle endurance. Aust J Physiother, 1998, 44: 201-207. [Medline]

4) Jull G, Falla D, Treleavan J, et al.: A therapeutic exercise approach for cervical disorders. In: Boyling JD, Jull G, eds. Grieve's Modern Manual Therapy, The Vertebral Coloumn. Edingburgh: Churchill Livingstone, 2004.

5) Jeon JY, Ju SB, Jeong HJ: The effect of cervical stabilizing exercises in the standing position and the supine position on deep neck muscle strength and endurance. J Phys Ther Sci, 2012, 24: 423-425. [CrossRef]

6) Gong WT, Park GD, Lee JH: Effects of longus colli muscle massage on the cervical extension range of motion of adults. J Phys Ther Sci, 2012, 24: 999-1001. [CrossRef]

7) Clay JH, Pounds DM: Basic Clinical Massage Therapy: Intergrating anatomy and treatment. Philadelphia: Lippincott Williams \& Wilkins, 2006, pp 92-110.

8) Jull G, Falla D, Hodges P, et al.: Cervical flexor muscle retraining: physiological mechanisms of efficacy. Paper presented at 2nd international conference on movement dysfuction. Edingburgh, 2005. 